\title{
Function and Length Scale Integration Study in Emerging MST-Based Products
}

\author{
Samuel Bigot, Stefan Dimov, and Roussi Minev \\ The Manufacturing Engineering Centre \\ Cardiff University, Queen's Buildings, The Parade, Newport Road \\ Cardiff CF24 3AA, United Kingdom
}

\begin{abstract}
This paper discusses the issues typically occurring when integrating, into a single product, functions that can only be realised by employing different length scale features (macro, meso, micro and down to nano). Following the responses of a survey performed on European research projects, a trend for replacing assembly steps with multi-scale processing of single components is highlighted. Two main issues emerging from the implementation of such approach are then discussed. The first one relates to the concurrent design of materials properties and manufacturing processes. The second focuses on the need for new production approaches and organisational models applied by companies.
\end{abstract}

Keywords: Multiple-scale, functional integration, length scale integration, material design, organisation models, micro and nano manufacturing.

\section{Introduction}

New innovative products rely more and more on the integration of multiple functions in as small as possible enclosures, and thus on miniaturization that simultaneously broaden their functionality. Such products encapsulate in a single container [1] various functions emerging from different research field, for instance nanoelectronics, microsensors, micro and/or nano actuators or microfluidic, and would generally incorporate different length scale features, from the millimeter to the nanometer range. The concerted efforts of designers and manufacturing specialists to achieve such Function and Length Scale Integration (FLSI) can be justified by the numerous advantages that the adoption of this product development philosophy offers as reductions in cost, size, material usage and power consumption.

The FLSI challenge, which can be shortly defined as the integration of a range of functional features with different length scales into a single product, generally relies on emerging micro-assembly technologies. Alternatively, due to the intrinsic relationship between functional features and machined structures, the incorporation of various functions can sometimes be performed directly, without assembly, in a single component using emerging micro and nano structuring technologies. In any case, this is a difficult task due to the necessity to perform such integration reliably and cost effectively, at nano through micro to meso meter scales. 
There are already many emerging product ideas and concepts based on FLSI, which are often a result of multidisciplinary R\&D programmes. For instance, the following products are good examples of innovative applications of this design and manufacturing approach: (i) the polymer-based lab-on-chip platform (Figure 1.a) for protein detection in point-of-care applications developed in the European FP6 project SEMOFS [2], which aimed at integrating active optical components like a planar surface plasmon resonance sensors with a microfluidic system (including actuators); (ii) a contact lens encapsulating micron-scale metal interconnects in a biocompatible polymer (Figure 1.b) that includes light emitting diodes [3]. The described third generation contact lens is opening the door to a wide range of exiting new consumer products, such as a see-through display that could be both remotely powered and controlled via a wireless link with potential applications in gaming, training, and manufacturing [4].
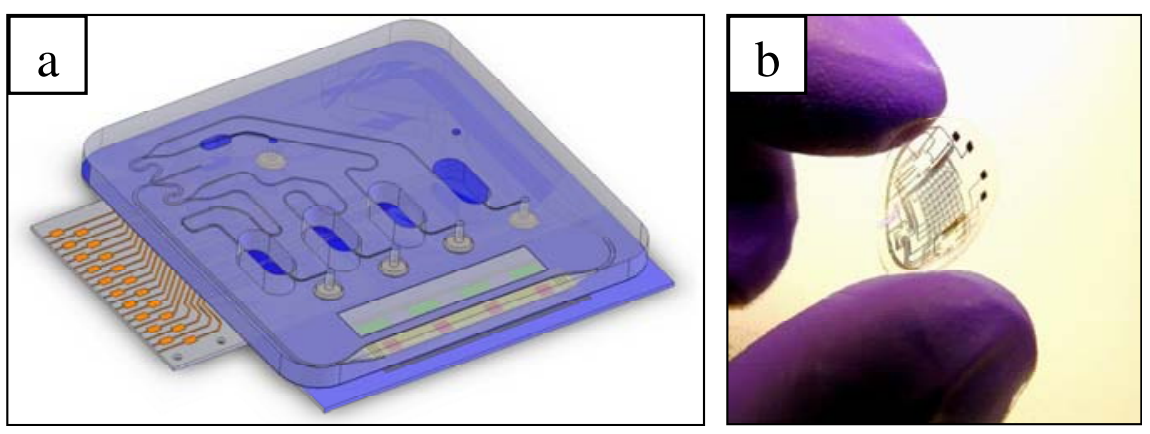

Fig. 1. a) Low-cost lab-on-chip b) Multifunction contact lens [3]

A common aspect in all these products is that the integration of various functions generally requires the latest state-of-the-art technologies, and in many cases the development of new manufacturing methods and chains of processes for incorporating different length-scale structures/features in a single component or the use of novel assembly techniques. The structure of the paper is as follows.

Based on a survey, the first section of the paper discusses the main integration methods used during the development of new MST based products. A trend for replacing assembly steps with multi-scale processing of single components is highlighted and the two main issues emerging from the implementation of such approach are then discussed in the last two sections.

\section{Integration: From Assembly to Single Component Processing}

A survey of 88 European research and development projects was conducted. All projects were focused on the development of MNT-based products and micro/nano manufacturing technologies that underpin their production while 54 of them involved companies as partners. One of the main objectives of this survey was to identify typical issues occurring when designing and manufacturing products incorporating functional features with different length scales. In this context, it was reported that 75 of 
the surveyed projects targeted the production of micro/nano components, while for 48 of them it was stated that the integration of micro and nano structures represented a significant problem.

The importance of integration using an assembly stage in micro and nano manufacturing was clearly supported by the results of the conducted survey where for $52 \%$ of the projects an assembly step was used in some way as part of the FLSI process, and it was the only FLSI process used in approximately $31 \%$ of the projects. Assembly is indeed a key stage in the fabrication of a product, which may require the use of various processes to integrate components manufactured separately due to different technical reasons, e.g. complexity, different material properties, capabilities restrictions and/or incompatibility of the available manufacturing processes [5]. 12 of the 44 FLSI issues reported were related to an assembly step. Particular concerns were the cost and reliability of bounding techniques used when dealing with micro components, and also dimensional accuracy of the components to be assembled.

For many economical and technical reasons and taking into account general rules from Design for Manufacture [6] it is generally better to achieve the required functionality of component or products using the minimum number of parts and thus to minimise the assembly steps in achieving FLSI in products, if not possible to eliminate them all together. This generic Design for Manufacture consideration was confirmed in the survey where $40 \%$ of the projects with as objective the development of new products use assembly as the sole procedure for FLSI while this is the case only with $15 \%$ of the projects targeting improvements of existing products. For such projects in most cases the improvements are sought / achieved through the use of a chain of multiple processes working on a single component, which suggests that this solution is preferred in the attempts to eliminate and/or minimise the assembly operations. By applying this approach towards FLSI, features with different length scales are machined directly on a single component. To achieve this, a sequence of compatible and complementary multidisciplinary processes are used, most of which can be classified as removal, deposition, deformation or replication ones. However, in implementing this approach new integration issues arise. Materials should be compatible with the set of micro/nano manufacturing technologies used in a process chain and specific organisational approaches are likely to be required to face the interdisciplinary issues and high capital investments linked with micro manufacturing.

\section{Concurrent Material and Process Design}

When integrating different length scale features, the microstructure of the materials that will undergo structuring should be optimised taking into account their specific machining response to various meso/micro/nano manufacturing technologies used in combinations while meeting the end product property requirements. This can be difficult to achieve as these requirements might differ significantly or even contradict each other.

Evidences of the need for the development of specifically tailored materials for micro-manufacturing are emerging, such as in the R\&D programme on "Integrated Development of Materials and Processing Technology for High Precision Components" 
funded by NEDO in Japan [7] and the findings of the "International Assessment of Research and Development in Micro-manufacturing" in the USA [8].

There are a number of material deposition and refinement technologies (solidsolid, liquid-solid, vapour-solid, etc.), that can be readily applied to design/tune the material microstructure, and thus achieve a step change in their machining response. Examples of such technologies are Physical Vapour Deposition (PVD), Chemical Vapour Deposition (CVD), melt quenching, Electrochemical Deposition (ECD) or severe plastic deformation (SPD), with which it is possible to produce 'nano metals' that have more appropriate amorphous (glassy) structure or grain sizes down to sub$100 \mathrm{~nm}$ compared to a typical grain size of $100 \mu \mathrm{m}$ in traditional metals.

Two recently completed feasibility studies illustrate the potential of such integrated approach for material and process design [9,10]. In the first study, by designing/optimising a $\mathrm{Ni}$ workpieces microstructure (e.g. grain size which controls the surface grain anisotropy) and the Focus Ion Beam (FIB) milling technology (ion fluence which controls the process dynamics) simultaneously it was possible to identify processing and performance windows that "overlap", and thus lead to synergetic effects on achievable feature resolution and surface integrity. The second study involved micro milling of two Al alloy workpieces that were metallurgically and mechanically modified, respectively. The result showed that by refining the material microstructure through SPD it is possible to achieve drastic improvements, more than three times, of surface roughness of thin features, $20 \mu \mathrm{m}$ ribs, in micro-components [10]. In both cases, the synergetic material and process development led to a "step change" in surface quality, dimensional accuracy and functional properties of the final product.

Ultimately, to properly implement a concurrent product, material and process design, it is necessary, together with the process design, to optimise the micro-structure of materials and thus to improve their processing response to one or even a set of component technologies in a process chain (Figure 13). This could be achieved by refining/tuning the bulk material to achieve a significantly better machining response with the majority of the manufacturing processes and/or by locally "tuning" the surface layer, with sub-mm thickness, which will undergo structuring with other complementary manufacturing processes.

Using such material design approach for FLSI gives almost limitless structuring possibilities. The main issues are the production cost which can increase significantly due to the complexity and wide range of capital-intensive state-of-the-art technologies involved. However, with an appropriate business organisation, discussed in next section, such approach would be particularly applicable for the fabrication of replication tools, e.g. for injection moulding, glass moulding, hot embossing, roll to roll imprinting, stamping and coining, with which very large batches of parts can be produced, more than 100000 per batch, and thus making the implementation of this approach cost effective.

\section{New Production Approaches}

Many SME's position themselves as partners of large companies and cooperate with them in a made-to-order fashion. Such a model gives little room, if any, for FLSI issues to be properly taken into account in the product design and manufacture stages, 
because they would necessitate the manufacturers to dynamically and cost-effectively integrate, optimise, configure, simulate, restructure and control not only their available in house surface structuring systems but also their materials' supply networks and part designs, in a coordinated manner. There are attempts to address these difficulties, e.g. in the production of micro parts by micro injection moulding. In particular, some replication machine providers work very closely with tool makers to develop and sell "packages", replication machines together with the necessary tooling and even to optimise processing parameters to satisfy specific product requirements, rather than focusing on each component technology separately. This approach could be broadened to include the material providers in the development of such solutions, and thus to offer materials optimised for specific FLSI requirements.

To deal with the interdisciplinary issues related to FLSI and the high capital investments required, new organisational approaches such as distributed production systems have to be considered. This will allow companies to design and produce parts without actually having all the required equipment and expertise physically on site, avoiding costly capital investments and personnel training. This is reflected in the responses obtained for the surveyed projects, where the majority of project consortia took the form of value networks or horizontal value chains, especially when considering projects focusing on the development of new products. Distributed production is not new in itself. For example, the dental industry uses it for the manufacturing of crowns, while the tool industry for the manufacturing of drills. But, in all existing cases, only variations of predetermined shapes can be produced. In a FLSI distributed system any complex 3D geometry at meso, micro and nano scale would have to be achievable. Designing and producing such products would require a highly integrated organisational model, bringing together a wide range of expert organisations.

Models such as extended enterprises and more specifically virtual enterprises, which can be viewed as a temporary alliance of companies taking advantage of a market opportunity [11], would have to be considered. Such approach will allow companies to focus on their key competencies without having to realise the complete process chain in house.

\section{Conclusion}

The results of a survey performed on a portfolio of 88 European research projects highlighted a inclination to replace assembly steps with multi-scale processing of single components when design MNT-based products. According to the survey the preferred methods for addressing the FLSI issues when developing new products are still the assembly ones. Nevertheless a trend for replacing them with multi-scale processing solutions can be identified, especially when improving MNT-based products. The challenges associated with the implementation of such solutions were discussed, too. In particular, the need for a new concurrent process-material development approach was presented in order to take full advantage of machining capabilities offered by component technologies in process chains, and thus to create the necessary prerequisites for achieving FLSI. Finally, the necessity of new organisational approaches was discussed to address technical and investments challenges that have to be tackled in achieving FLSI, e.g. finding different innovative ways for implementing distributed production systems in the MNT context. 
Acknowledgments. The research reported in this paper is funded by the Engineering and Physical Sciences Research Council (GR/S75505) and the ERDF Programme "Supporting Innovative Product Engineering and Responsive Manufacture". Also, it was carried out within the framework of the EC FP6 Networks of Excellence, "MultiMaterial Micro Manufacture (4M): Technologies and Applications" and the "Innovative Production Machines and Systems (I*PROMS)".

\section{References}

1. Evoy, S., DiLello, N., Deshpande, V., Narayanan, A., Liu, H., Riegelman, M., Martin, B.R., Hailer, B., Bradley, J.-C., Weiss, W., Mayer, T.S., Gogotsi, Y., Bau, H.H., Mallouk, T.E., Raman, S.: Dielectrophoretic assembly and integration of nanowire devices with functional CMOS operating circuitry. Microelectronic Engineering 75, 31-42 (2004)

2. Nestler, J., Morschhauser, A., Hiller, K., Otto, T., Bigot, S., Auerswald, J., Knapp, H.F., Gavillet, J., Gessner, T.: Polymer Lab-on-Chip systems with integrated electrochemical pumps suitable for large scale fabrication. International Journal of Advanced Manufacturing Technologies (2009)

3. Ho, H., Saeedi, E., Kim, S.S., Shen, T.T., Parviz, B.A.: Contact lens with integrated inorganic semiconductor devices. In: Proceedings of the IEEE 21st International Conference on Micro Electro Mechanical Systems, pp. 403-406 (2008)

4. Parviz, B.A., Lingley, A.: Multipurpose integrated active contact lenses. SPIE Newsroom (2009), http: / / spie.org/×35114.xml?ArticleID=x35114

5. Hansen, H.N., Tosello, G., Gegeckaite, A., Arentoft, M., Marin, L.: Classification of assembly techniques for micro products. In: Proceedings of the 1st International Conference on Multi Material Micro Manufacture, Karlshrue, Germany, pp. 283-286 (2005)

6. Boothroyd, G., Dewhurst, P., Knight, W.A.: Product Design for Manufacture and Assembly. CRC Press, Boca Raton (2001)

7. Hayashi, K., Kiuchi, M.: Integrated Development of Materials and Processing Technology for High Precision Components. Sosei Kako Shinpojiumu 243, 1-11 (2005)

8. WTEC Panel: International Assessment of Research and Development in Micro manufacturing (2005), http: / / www . wtec . org / micromfg /

9. Li, W., Minev, R., Dimov, S., Lalev, G.: Patterning of Amorphous and Polycrystalline Ni78B14Si8 with a Focused Ion Beam. Applied Surface Science 253(12), 5404-5410 (2007)

10. Popov, K.B., Dimov, S.S., Pham, D.T., Minev, R.M., Rosochowski, A., Olejnik, L.: Micromilling: material microstructure effects. Proceedings of the Institution of Mechanical Engineers-Part B 220(11), 1807-1813 (2006)

11. Szegheo, O., Petersen, S.A.: Extended Enterprise Engineering - A Model-Based Framework. Concurrent Engineering 8(1), 32-39 (2000) 